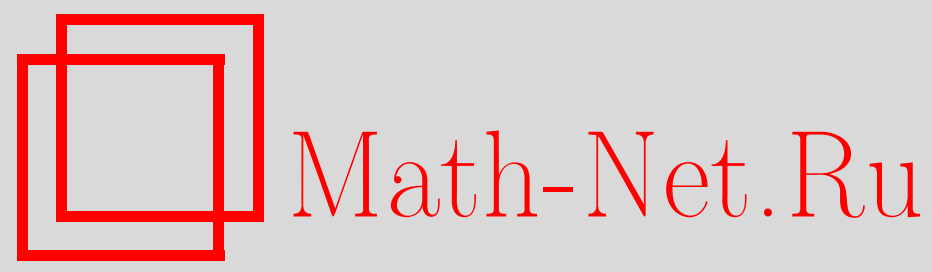

Ю. А. Комбаров, О минимальных схемах для линейных функций в некоторых базисах, Дискрет. матем., 2013, том 25, выпуск 1, 33-44

DOI: https://doi.org/10.4213/dm1219

Использование Общероссийского математического портала Math-Net.Ru подразумевает, что вы прочитали и согласны с пользовательским соглашением http://www . mathnet.ru/rus/agreement

Параметры загрузки:

IP : 34.229 .108 .108

26 апреля 2023 г., 16:55:35 


\title{
О минимальных схемах для линейных функций в некоторых базисах
}

\author{
() 2013 г. Ю. А. Комбаров
}

\begin{abstract}
В данной работе рассматриваются реализации линейных булевых функций схемами из функциональных элементов в базисе $\overline{5}$, состоящем из двухвходовых функциональных элементов, реализующих нелинейные функции, и инвертора. Вес двухвходового элемента в базисе 5 принимается равным единице, а вес инвертора - нулю.

В работе дано описание всех минимальных схем, реализующих линейные функции в базисе 5 , а именно, показано, что все такие схемы имеют определенный блочный вид.

Работа выполнена при финансовой поддержке Российского фонда фундаментальных исследований, проект 11-01-00508, и Программы фундаментальных исследований Отделения математических наук РАН «Алгебраические и комбинаторные методы математической кибернетики и информационные системы нового поколения», проект «Задачи оптимального синтеза управляющих систем».
\end{abstract}

В алгебре логики и в математической кибернетике один из наиболее изученных классов булевых функций составляют линейные функции

$$
f\left(x_{1}, \ldots, x_{n}\right)=x_{1} \oplus \ldots \oplus x_{n} \oplus c, \quad n \in \mathbf{N}, \quad c \in\{0,1\},
$$

символ $\oplus$ означает сложение по модулю два. Для этих функций, в частности, установлена сложность их реализации схемами из функциональных элементов [1] в некоторых базисах. В [2] установлено, что

$$
L\left(x_{1} \oplus \ldots \oplus x_{n} \oplus c\right)=4 n-4
$$

в базисе $\{x \& y, x \vee y, \bar{x}\}$ и

$$
L\left(x_{1} \oplus \ldots \oplus x_{n} \oplus c\right)=7 n-7
$$

в базисах $\{x \& y, \bar{x}\}$ и $\{x \vee y, \bar{x}\}$; здесь через $L(f)$ обозначается сложность реализации функции $f$, то есть наименьшее число функциональных элементов в схеме, реализующей $f$. Дальнейшие результаты в этом направлении таковы:

$$
L\left(x_{1} \oplus \ldots \oplus x_{n}\right)=4 n-4
$$

в базисе $\{\overline{x \& y}\}$ (см. [3]);

$$
L\left(x_{1} \oplus \ldots \oplus x_{n}\right)=4 n-4
$$

2 Дискретная математика, т.25 №1 
в базисе $\{x \rightarrow y, \bar{x}\}$ (см. [4]);

$$
L\left(x_{1} \oplus \ldots \oplus x_{n} \oplus 1\right)=4 n-4
$$

при четных $n$ и

$$
L\left(x_{1} \oplus \ldots \oplus x_{n}\right)=4 n-4
$$

при нечетных $n$ в базисе $\{\bar{x} \& y, \bar{x}\}$ (см. [5]). Для базиса $\{x \& y, x \vee y, \bar{x}\}$ известна структура всех минимальных схем, реализующих линейные функции. Соответствующий результат сформулирован в [6], а доказательство можно найти в [7].

В данной работе рассматриваются минимальные реализации функций

$$
\begin{aligned}
& l_{n}\left(x_{1}, \ldots, x_{n}\right)=x_{1} \oplus \ldots \oplus x_{n}, \\
& \bar{l}_{n}\left(x_{1}, \ldots, x_{n}\right)=x_{1} \oplus \ldots \oplus x_{n} \oplus 1, \quad n \geqslant 2,
\end{aligned}
$$

схемами в базисе

$$
Б=\left\{E_{1}, \ldots, E_{k}, E^{-}\right\},
$$

где $k$ - любое число из $\{1,2, \ldots, 6\}$, а элементы $E_{1}, \ldots, E_{k}$ - это различные двухвходовые функциональные элементы, реализующие любые $k$ функций из $\{x \& y, x \vee y, \bar{x} \& y, \bar{x} \vee y$, $x \& y \oplus 1, x \& y \oplus x \oplus y \oplus 1\}$. При подсчете сложности схем мы будем учитывать только двухвходовые элементы.

Пусть $S$ - схема в базисе $Б$. Число всех входящих в нее двухвходовых элементов будем называть сложностью схемы и обозначать $L(S)$. Будем говорить, что $S-$ минимальная схема для функции $f$, если $S$ реализует $f$, причем для любой другой схемы $S^{\prime}$, реализующей $f$, выполняется неравенство $L(S) \leqslant L\left(S^{\prime}\right)$, и среди всех схем, реализующих $f$ и имеющих сложность $L(S)$, схема $S$ содержит наименьшее число элементов.

Пусть $V$ - выход функционального элемента $E_{1}$ или вход схемы, а $W-$ вход функционального элемента $E_{2}$. Будем говорить, что между элементами $E_{1}$ и $E_{2}$ (или между соответствующим входом схемы и $E_{2}$ ) находится инвертор $E^{-}$, если $V$ подается на вход элемента $E^{-}$, а выход инвертора $E^{-}$подается на $W$ (и, быть может, на какие-либо входы функциональных элементов, отличные от $W$ ). Будем говорить, что $V$ связан с $W$, если $V$ подается на $W$ или если между $E_{1}$ (или входом схемы) и $E_{2}$ есть инвертор. Будем называть двухвходовой функциональный элемент $E$ верхним, если оба его входа связаны со входами схемы. Такой функциональный элемент существует в любой схеме по соображениям монотонной нумерации элементов в схеме [8].

Будем говорить, что в схеме $S$ можно выделить стандартный блок, если в ней можно выбрать две вершины (вершинами в данном случае будем называть входы схемы или выходы элементов) $V_{1}, V_{2}$ и три двухвходовых функциональных элемента $E_{1}, E_{2}$ и $E_{3}$ так, что вершина $V_{1}$ связана со входами элементов $E_{1}$ и $E_{2}$, вершина $V_{2}$ связана со входами элементов $E_{1}$ и $E_{2}$, выход элемента $E_{1}$ связан со входом элемента $E_{3}$, выход элемента $E_{2}$ связан со входом элемента $E_{3}$, выходы элементов $E_{1}$ и $E_{2}$ не связаны со входами никаких других элементов схемы $S$ (кроме $E_{3}$ ), при подаче на вершины $V_{1}$ и $V_{2}$ функций $x_{1}$ и $x_{2}$ на выходе элемента $E_{3}$ реализуется функция $x_{1} \oplus x_{2}$ или $x_{1} \oplus x_{2} \oplus 1$, и выход всякого инвертора, находящегося между вершиной $V_{1}$ и входами элементов $E_{1}$ и $E_{2}$, между вершиной $V_{2}$ и входами элементов $E_{1}$ и $E_{2}$, и между выходами элементов $E_{1}, E_{2}$ и входами элемента $E_{3}$, подается ровно на один вход одного функционального элемента. Подсхему, состоящую из элементов $E_{1}, E_{2}, E_{3}$, а также всех инверторов, находящихся между вершиной $V_{1}$ и входами элементов $E_{1}$ и $E_{2}$, между вершиной $V_{2}$ и входами 
элементов $E_{1}$ и $E_{2}$, и между выходам элементов $E_{1}, E_{2}$ и входами элемента $E_{3}$ будем называть стандартным блоком. Вершины $V_{1}$ и $V_{2}$ будем называть входными вершинами стандартного блока.

Стандартный блок $B$ в схеме $S$ будем называть верхним, если обе его входные вершины являются входами схемы. Применительно к верхнему стандартному блоку $B$ схемы $S$ введем операцию стандартной редукции, заключающуюся в том, что одна из входных вершин блока $B$ (и схемы $S$ ) отождествляется с выходом блока $B$ и сохраняется как вход редуцированной схемы $S^{\prime}$ (получающейся из $S$ ), а вторая входная вершина и все элементы блока $B$ удаляются из $S$.

Несложно заметить, что из определения стандартной редукции вытекают следующие утверждения.

Лемма 1. Если схема $S$ реализует линейную функцию $n$ переменных, $n \geqslant 2$, и к ней применяется операция стандартной редукции, то редуц̧ированная схема $S^{\prime}$ реализует линейную функиию $n-1$ переменной.

Лемма 2. Если в редуцированной схеме $S^{\prime}$ можно выделить некоторые стандартные блоки $B_{1}, \ldots, B_{k}$, то эти же блоки можно выделить и в исходной схеме $S$.

В следующей теореме находится сложность реализации линейных функций в базисе $\overline{\text {. }}$.

Теорема 1. Сложность реализации функциий

$$
\begin{aligned}
l_{n} & =l_{n}\left(x_{1}, x_{2}, \ldots, x_{n}\right) \\
& =x_{1} \oplus x_{2} \oplus \ldots \oplus x_{n}, \\
\bar{l}_{n} & =x_{1} \oplus x_{2} \oplus \ldots \oplus x_{n} \oplus 1, \quad n \geqslant 2,
\end{aligned}
$$

в базисе Б составляет $3 n-3$.

Доказательству теоремы предпошлем несколько вспомогательных утверждений.

Лемма 3. Для любого натурального п справедливы неравенства

$$
L\left(l_{n}\right) \leqslant 3 n-3, \quad L\left(\bar{l}_{n}\right) \leqslant 3 n-3 .
$$

Доказательство. Неравенства доказываются индукцией по $n$, поскольку

$$
\begin{aligned}
l_{n} & =l_{n}\left(x_{1}, x_{2}, \ldots, x_{n}\right) \\
& =l_{n-1}\left(x_{1}, x_{2}, \ldots, x_{n-1}\right) \oplus x_{n}, \\
\bar{l}_{n} & =\overline{x_{1} \oplus x_{2} \oplus x_{3} \oplus \ldots \oplus x_{n}},
\end{aligned}
$$

а линейная функция от двух переменных может быть реализована схемой в базисе $Б$ с весом, равным трем (эта схема является одним из стандартных блоков).

Нижняя оценка будет следовать из леммы 7.

Будем считать, что на входы схем разрешается подавать константы 0 и 1 . Будем говорить, что константа забивает двухвходовый функциональный элемент по левому (правому) входу или является забивающей для элемента по левому (правому) входу, если при подаче этой константы на соответствующий вход данного элемента на его выходе получаем константу. Несложно видеть, что для каждого из шести двухвходовых элементов, которые могут входить в базис $Б$, такие константы существуют: для обоих входов элементов $x \& y$ и $x \& y \oplus 1$ это ноль, для обоих входов элементов $x \vee y$ и $x \& y \oplus x \oplus y \oplus 1$ 
- один, для левого входа элемента $\bar{x} \& y$ и правого входа элемента $\bar{x} \vee y-$ это один, и для правого входа элемента $\bar{x} \& y$ и левого входа элемента $\bar{x} \vee y-$ это ноль. Заметим также, что если на вход двухвходового функционального элемента подается константа, не забивающая его, то на выходе получаем функцию $\phi$ или функцию $\bar{\phi}$, где $\phi-$ функция, подающаяся на другой вход элемента.

Лемма 4. Пусть $S$ - схема в базисе Б, реализующая функцию $\phi, ~ a E-$ элемент в ней. Тогда если Е реализует булеву константу с, то мы можем удалить $E$ и получить схему, реализуюшую ту же функцию ф.

Доказательство. После удаления $E$, достаточно подать на входы элементов, на которые подавался выход элемента $E$, вход схемы, отвечающий константе $c$.

Лемма 5. Пусть $S-$ схема в базисе Б, реализующая функичю $\phi, ~ a E-$ элемент в ней. Тогда, если на вход элемента Е подается константа, то мы можем удалить Е или заменить Е на инвертор, получив схему, реализующую ту же функцию ф.

Доказательство. Пусть $\psi-$ функция, подаваемая на другой вход элемента $E$. Если на $E$ подается забивающая константа, воспользуемся леммой 4. В противном случае $E$ реализует $\psi$ или $\bar{\psi}$. В первом случае удалим $E$, подав $\psi$ на элементы, на которые подавался выход элемента $E$, во втором заменим $E$ на инвертор, на который подается функция $\psi$.

Лемма 6. Пусть $S-$ схема в базисе Б, реализующая функциию $\phi, E-$ элемент в ней, $x$ - одна из переменных, подаюшихся на вход схемы. Тогда если Е реализует функцию 廿, тождественно равную х или $\bar{x}$, то мы можем удалить Е или заменить $E$ на инвертор, получив схему, реализуюшую ту же функиию ф.

Доказательство. Если $E$ реализует функцию $x$, то удалим $E$ и подадим на входы элементов, на которые подавался выход элемента $E$, вход схемы, соответствующий переменной $x$, а если $E$ реализует $\bar{x}$, то заменим $E$ на инвертор, на вход которого подается вход схемы, соответствующий переменной $x$.

Лемма 7. Если $S_{n}, n \geqslant 2$, - минимальная схема, реализующзая функциюю $l_{n}$ или $\bar{l}_{n}$ в базисе Б, то из схемы $S_{n}$ можно удалить не менее трех двухвходовых элементов и, возможно, добавить несколько инверторов так, что получившаяся схема $S_{n-1}$ будет реализовывать одну из функций $l_{n}, \bar{l}_{n}$.

Доказательство. Рассмотрим несколько случаев.

1. Пусть в схеме $S_{n}$ существует двухвходовой функциональный элемент $E$, оба входа которого связаны с одним и тем же входом схемы, соответствующим некоторой переменной. Тогда $E$ реализует функцию не более чем от одной переменной, а значит, этот элемент можно удалить из схемы или заменить на инвертор, воспользовавшись леммами 4 или 6, при этом сложность схемы уменьшится на 1 , а реализуемая ею функция не изменится. Это противоречит предположению о минимальности схемы. При рассмотрении последующих случаев будем считать, что в схеме нет элементов, подобных $E$.

2. Пусть в схеме $S_{n}$ вход, соответствующий некоторой переменной $x$, связан со входом ровно одного двухвходового функционального элемента $E$. Обозначим $\phi$ функцию, подающуюся на другой вход элемента $E$. Покажем, что случай 2 невозможен. Рассмотрим следующие подслучаи. 
2.1. Если $\phi$ не является константой, то поскольку $\phi$, очевидно, не зависит от $x$, всегда можно подобрать значения остальных переменных так, чтобы функция $\phi$ принимала постоянное значение, забивающее элемент $E$ (по входу, на который подается эта функция). Тогда переменная $x$ забивается остальными переменными, что для линейной функции невозможно.

2.2. Пусть теперь функция $\phi$ является константой. Если $\phi$ забивает элемент $E$, то, как и в случае 2.1 , переменная $x$ забивается остальными переменными. Поэтому предположим, что константа $\phi$ не забивает элемент $E$. Тогда $E$ можно удалить, воспользовавшись леммой 5, что входит в противоречие с условием минимальности схемы $S_{n}$.

3. Пусть каждый вход схемы связан со входами более чем одного двухвходового элемента. Предположим, что вход, соответствующий переменной $x$, связан с входом двухвходового элемента $E_{1}$ и с входом двухвходового элемента $E_{2}$. Ни один из элементов $E_{1}, E_{2}$ не является выходным и в силу минимальности $S_{n}$ выход каждого из элементов $E_{1}, E_{2}$ должен быть связан с входом двухвходового элемента (если элемент $E$ не является выходным в схеме и его выход не подается на входы других элементов схемы, то такой элемент $E$ можно удалить из схемы). Так как схема не содержит циклов, то выход хотя бы одного из этих элементов (без ограничения общности, элемента $E_{2}$ ) связан с входом некоторого двухвходового элемента $E_{3}$, отличного от элементов $E_{1}, E_{2}$. Снова подаем вместо $x$ константу такую, что на вход элемента $E_{2}$ поступает константа, забивающая $E_{2}$. Тогда элемент $E_{2}$ реализует константу, на входы элементов $E_{1}$ и $E_{3}$ подаются константы, поэтому элементы $E_{1}, E_{2}$ и $E_{3}$ можно удалить или заменить на инверторы (по леммам 4 и 5). Таким образом, получаем схему $S_{n-1}$, реализующую функцию $l_{n-1}$ или $\bar{l}_{n-1}$, причем сложность $S_{n-1}$ в базисе $Б$ удовлетворяет условию

$$
L\left(S_{n}\right)=L\left(S_{n-1}\right)+3 .
$$

Лемма 7 доказана.

Лемма 8. Для схем в базисе Б справедливо равенство

$$
L\left(l_{2}\right)=L\left(\bar{l}_{2}\right)=3 .
$$

Доказательство. Покажем, что ни $l_{2}$, ни $\bar{l}_{2}$ нельзя реализовать схемой, в которой только два двухвходовых элемента. Предположим обратное. Отбрасывая при необходимости инвертор, расположенный между выходом схемы и одним из этих элементов, получаем схему, которая также реализует $l_{2}$ или $\bar{l}_{2}$, и выходной элемент которой уже не является инвертором. Следовательно, один из входов выходного элемента связан со входом схемы, соответствующим некоторой переменной, которая, очевидно, забивает другую переменную. Полученное противоречие доказывает лемму 8.

Доказательство теоремь 1. Верхняя оценка следует из леммы 3. Нижняя оценка получается индукцией по $n$ с использованием леммы 8 в основании индукции и леммы 7 при индуктивном переходе.

Следующая теорема 2 дает описание минимальных схем из функциональных элементов в базисе 5 , реализующих функции $l_{2}$ и $\bar{l}_{2}$.

Теорема 2. Любую минимальную схему в базисе Б, реализуюшую $l_{n}$ или $\bar{l}_{n}, n \geqslant 2$, можно разбить на $n-1$ непересекающихся стандартных блоков.

Утверждение теоремы 2 следует из леммы 9. 
Лемма 9. Любая минимальная схема $S_{n}, n \geqslant 2$, в базисе Б, реализующая

$$
l_{n}=x_{1} \oplus x_{2} \oplus \ldots \oplus x_{n}
$$

или

$$
\bar{l}_{n}=x_{1} \oplus x_{2} \oplus \ldots \oplus x_{n} \oplus 1
$$

допускает операцию стандартной редукции.

Доказательство. Всюду при доказательстве леммы для краткости мы будем писать “вход $(x)$ ” вместо “вход, соответствующий переменной $x$ ”.

Возможны только четыре случая.

1. В схеме $S_{n}$ существует вход $(x)$, связанный с двумя входами какого-то двухвходового элемента $E$. Невозможность случая 1 была показана при доказательстве леммы 7.

2 . В схеме $S_{n}$ есть вход $(x)$, связанный со входом ровно одного двухвходового элемента $E$. Невозможность случая 2 также была показана в доказательстве леммы 7.

3. В схеме $S_{n}$ есть вход $(x)$, связанный со входами трех двухвходовых функциональных элементов $E_{1}, E_{2}, E_{3}$. Выход хотя бы одного из этих элементов (без ограничения общности, пусть это будет выход элемента $E_{1}$ ) связан со входом двухвходового функционального элемента $E_{4}$. Подадим на вход $(x)$ такую константу, что на соответствующий вход $E_{1}$ подается забивающая константа. Тогда элемент $E_{1}$ реализует константу, и, следовательно, его можно удалить. При этом на элементы $E_{2}, E_{3}$ и $E_{4}$ также подается константа, что означает, что эти элементы можно удалить (по лемме 5), получив, таким образом, схему $S_{n-1}$, реализующую линейную функцию от $n-1$ переменной $l_{n-1}$ или ее отрицание $\bar{l}_{n-1}$ в базисе $Б$. Сложность схемы $S_{n}$ по теореме 1 равна $3 n-3$, поэтому сложность схемы $S_{n-1}$ будет равна $3 n-7$ (поскольку при переходе от $S_{n}$ к $S_{n-1}$ удаляется четыре двухвходовых элемента), что противоречит теореме 1 . Итак, случай 3 невозможен.

4. Каждый вход схемы $S_{n}$ связан ровно с двумя входами (ровно двух элементов). Разбиение случая 4 на подслучаи представлено в таблице на рис. 1 (для некоторых клеток таблицы условия уточняются в тексте).

4.1. Пусть входы верхнего элемента $E_{1}$ связаны со входами схемы $\left(x_{1}\right)$ и $\left(x_{2}\right)$. Пусть переменная $x_{2}$ подается также на вход элемента $E_{2}$, другой вход которого связан с выходом двухвходового элемента $E_{3}$ (см. рис. 2). Пусть $E_{4}-$ отличный от $E_{1}$ двухвходовой элемент, вход которого связан со входом $\left(x_{1}\right)$. Подадим на вход $\left(x_{1}\right)$ такую константу, что на соответствующий вход $E_{1}$ подается забивающая константа. Пусть после этого на тот вход $E_{2}$, который связан с выходом $E_{3}$, подается функция $\phi$. Так как схема не содержит циклов, $\phi$ может зависеть только от переменных $x_{3}, \ldots, x_{n}$.

4.1.1. Пусть функция $\phi$ не является константой. Тогда можно подобрать значения переменных $x_{3}, x_{4}, \ldots, x_{n}$ так, чтобы $\phi$ обращалась в забивающую константу для элемента $E_{2}$. Но это означает забиваемость переменной $x_{2}$ остальными переменными, что для линейной функции невозможно.

4.1.2. Пусть функция $\phi$ является константой.

4.1.2.1. Пусть элементы $E_{1}$ и $E_{3}$ совпадают. Если элемент $E_{4}$ является выходным, то переменная $x_{1}$ забивает все остальные переменные, что невозможно для линейной функции. Поэтому элемент $E_{4}$ не является выходным. Тогда существует двухвходовой элемент $E_{5}$, с входом которого связан выход $E_{4}$ (см. рис. 3 ). Подадим на вход $\left(x_{1}\right)$ такую константу, 


\section{4. В схеме $S_{n}$ каждый вход связан ровно с двумя входами (ровно двух двухвходовых элементов).}

\section{1. В схеме существует верхний} элемент $\mathrm{E}_{1}$, входы которого связаны со входами (х1) и (х2). Вход (х2) связан со входом элемента $\mathrm{E}_{2}$, другой вход $\mathrm{E}_{2}$ связан с выходом двухвходового элемента $\mathrm{E}_{3}$.

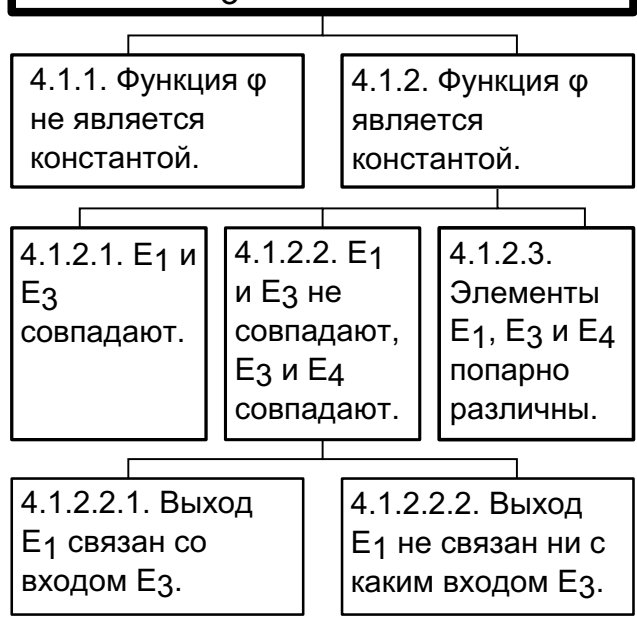

4.2. Все входы схемы, связанные со входами верхних элементов связаны со входами лишь верхних элементов.

4.2.1. В схеме существует верхний элемент $\mathrm{E}_{1}$, чьи входы связаны со входами схемы (х1) и (х2), причем входы (х1) и (х2) также связаны со входами двух различных двухвходовых функциональных элементов.

4.2.2.1. Выходы элементов $\mathrm{E}_{1}$ и $\mathrm{E}_{2}$ связаны со входами различных двухвходовых элементов Е3 и Е4.
4.2.2. Все входы схемы, всязанные с верхними элементами, разбиваются на пары, такие, что входы в паре связаны со входами одних и тех же двух элементов. $\mathrm{E}_{1}$ и $\mathrm{E}_{2}$ - одна из таких пар.

4.2.2.2. Выходы элементов $\mathrm{E}_{1}$ и $\mathrm{E}_{2}$ связаны со входами двухвходового функционального элемента Е3.

Рис. 1.

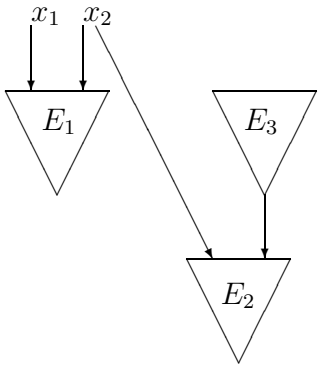

Рис. 2. 


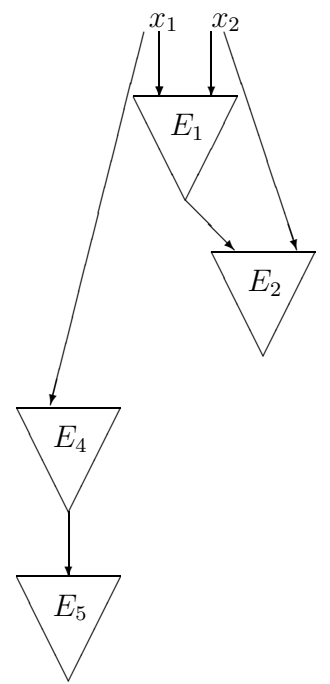

Рис. 3.

что на соответствующий вход $E_{4}$ подается забивающая константа. Тогда $E_{4}$ реализует константу, на $E_{5}$ подается константа, и кроме того $E_{1}$ реализует или константу, или $x_{2}$, или $\bar{x}_{2}$. Но тогда и $E_{2}$ реализует или константу, или $x_{2}$, или $\bar{x}_{2}$. Таким образом, все четыре элемента можно удалить или заменить на инверторы. Получаем противоречие с теоремой 1 .

4.1.2.2. Пусть элементы $E_{1}$ и $E_{3}$ не совпадают, а элементы $E_{3}$ и $E_{4}$ совпадают.

Элемент $E_{1}-$ не выходной, в противном случае $x_{1}$ забивала бы остальные переменные. Значит, его выход связан с входом какого-либо двухвходового элемента.

4.1.2.2.1. Пусть выход $E_{1}$ связан с входом $E_{3}$ (см. рис. 4). В этом случае выход элемента $E_{1}$ связан с входом лишь элемента $E_{3}$; если бы выход $E_{1}$ был также связан с входом некоторого элемента $E_{5}$, мы могли бы удалить четыре элемента, подав на вход $\left(x_{2}\right)$ такую константу, что на соответствующий вход $E_{1}$ подается забивающая константа. Аналогично, выход элемента $E_{3}$ связан лишь с входом элемента $E_{2}$. Но тогда переменная $x_{2}$ забивает переменную $x_{1}$ при подаче на вход $\left(x_{2}\right)$ такой константы, что на соответствующий вход элемента $E_{2}$ подается забивающая константа, что невозможно для линейной функции.

4.1.2.2.2. Пусть выход элемента $E_{1}$ связан с входом двухвходового функционального элемента $E_{5}$, отличного от $E_{1}, E_{2}, E_{3}$ (см. рис. 5). Напомним,что мы можем подать на вход $\left(x_{1}\right)$ такую константу, что на соответствующий вход $E_{1}$ будет подана забивающая константа, а $E_{3}$ будет выдавать функцию $\phi$, равную константе. Тогда $E_{1}$ и $E_{3}$ реализуют константы, а на $E_{2}$ и $E_{5}$ подаются константы, и эти четыре элемента можно удалить по леммам 4 и 5 , что противоречит минимальности схемы.

4.1.2.2.3. Пусть элементы $E_{1}, E_{3}$ и $E_{4}$ попарно различны. Напомним, что на вход $E_{1}$, связанный с входом $\left(x_{1}\right)$, можно подать такую константу, что на вход $E_{1}$ будет подана забивающая константа, а $E_{3}$ будет реализовывать функцию $\phi$ или $\bar{\phi}$, равную константе, то есть оба этих элемента будут реализовывать константы. Тогда и на $E_{4}$ и на $E_{2}$ также будут поданы константы (см. рис. 6). Значит, все рассматриваемые элементы можно будет удалить или заменить инверторами. Снова получаем противоречие с теоремой 1. 


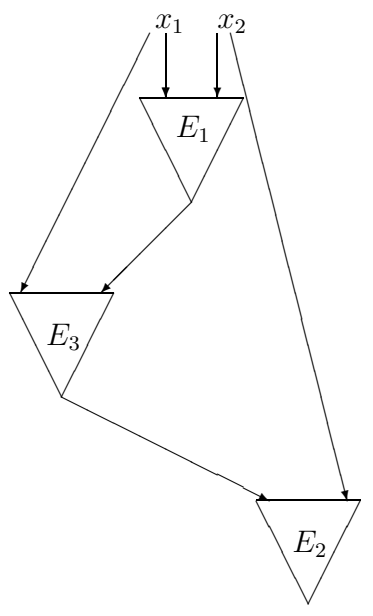

Рис. 4.

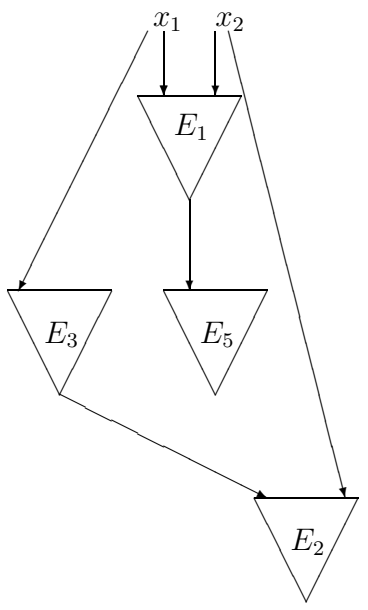

Рис. 5.

4.2. Пусть все входы схемы, связанные с входами верхних элементов, связаны с входами только верхних элементов (этот подслучай является отрицанием подслучая 4.1).

4.2.1. Предположим, что существуют входы схемы $\left(x_{1}\right)$ и $\left(x_{2}\right)$, связанные с входами элемента $E_{1}$, а также с входами различных элементов $E_{2}$ и $E_{3}$. В таком случае элементы $E_{2}$, $E_{3}$ - верхние и их входы связаны еще и с входами $\left(x_{i}\right),\left(x_{j}\right)$, отличными от входов $\left(x_{1}\right)$, $\left(x_{2}\right)$ (см. рис. 7). Подадим на вход $\left(x_{2}\right)$ такую константу, что на соответствующий вход $E_{1}$ подается забивающая константа, а на вход $\left(x_{i}\right)$ такую константу, что на соответствующий вход $E_{2}$ подается забивающая константа. Таким образом, переменная $x_{1}$ забивается переменными $x_{2}$ и $x_{i}$, что невозможно для линейной функции.

4.2.2. Пусть все входы схемы, соединенные с верхними элементами, разбиваются на пары, такие, что входы в каждой паре связаны с входами одних и тех же двух элементов. Рассмотрим одну такую пару входов, соответствующих переменным $x_{1}$ и $x_{2}$ и связанных 


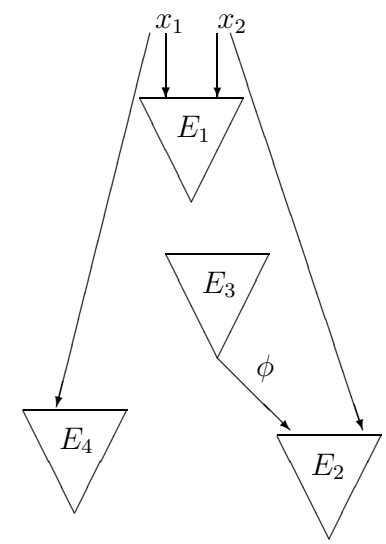

Pис. 6.

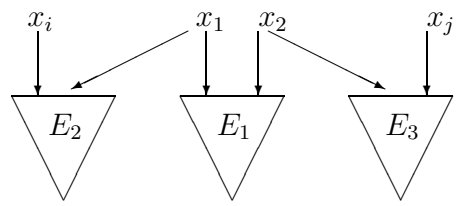

Рис. 7.

с входами элементов $E_{1}$ и $E_{2}$ (этот подслучай является отрицанием подслучая 4.2.1).

4.2.2.1. Предположим, что выходы элементов $E_{1}$ и $E_{2}$ связаны со входами различных двухвходовых элементов $E_{3}$ и $E_{4}$ (см. рис. 8).

Заметим, что выход $E_{1}$ связан с входом лишь элемента $E_{3}$. В самом деле, если выход $E_{1}$ связан еще и с входом некоторого элемента $E_{5}$, то, подав на вход $\left(x_{1}\right)$ такую константу, что на соответствующий вход $E_{1}$ подается забивающая константа, можно удалить элементы $E_{1}, E_{2}, E_{3}$ и $E_{5}$, что противоречит теореме 1 . Аналогично, выход элемента $E_{2}$ связан с входом лишь элемента $E_{4}$. Пусть на другой вход элемента $E_{3}$ подается функция $\phi$, а на другой вход элемента $E_{4}$ подается функция $\psi$. Так как схема не содержит циклов, то либо $\phi$, либо $\psi$ не зависит от $x_{1}$ и $x_{2}$. Без ограничения общности будем считать, что это

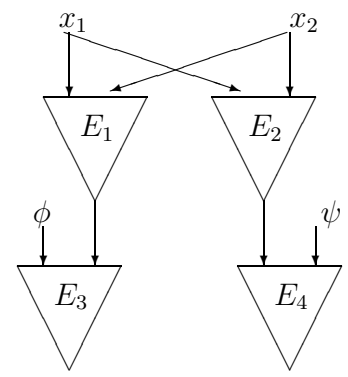

Рис. 8. 


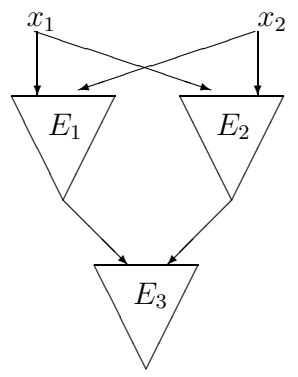

Рис. 9.

функция $\psi$. Если бы функция $\psi$ была константой, можно бы было удалить элемент $E_{4}$, что будет противоречить минимальности схемы. Поэтому функция $\psi$ не является константой. Подадим на вход $\left(x_{1}\right)$ такую константу, что на соответствующий вход $E_{1}$ подается забивающая константа, и подберем значения переменных $x_{3}, x_{4}$ так, чтобы функция $\psi$ превратилась в забивающую константу для $E_{4}$. Таким образом, переменная $x_{2}$ забивается остальными переменными, что невозможно.

4.2.2.2. Пусть выходы $E_{1}$ и $E_{2}$ связаны со входами одного элемента $E_{3}$ (см. рис.9).

Выходы элементов $E_{1}$ и $E_{2}$ связаны только с входами элемента $E_{3}$. В самом деле, если бы выход элемента $E_{1}$ был связан с входом некоторого элемента $E_{4}$, отличного от $E_{3}$, то подав на вход $\left(x_{1}\right)$ необходимую константу, такую, что на соответствующий вход $E_{1}$ подается забивающая константа, мы могли бы удалить четыре элемента $E_{1}, E_{2}, E_{3}$, $E_{4}$, что противоречит теореме 1 .

Элемент $E_{3}$ реализует функцию, существенно зависящую от двух переменных. В противном случае его можно было бы удалить или заменить на инвертор, что противоречило бы минимальности схемы. Более того, элемент $E_{3}$ реализует линейную функцию: ведь если бы он реализовывал какую-либо другую функцию от двух переменных, мы могли бы выбрать такое значение переменной $x_{1}$, что элемент $E_{3}$ реализовывал бы константу, а значит, выходное значение схемы не зависело бы от $x_{2}$, поскольку выходы элементов $E_{1}$ и $E_{2}$ связаны только с входами $E_{3}$.

Таким образом, в схеме можно выделить стандартный блок, содержащий элементы $E_{1}, E_{2}, E_{3}$, и схема допускает операцию стандартной редукции. Лемма 9 доказана.

Замечание 1. В [9] сформулирована следующая теорема.

Теорема 3. Все минимальные схемы в базисе

$$
E=\{x \rightarrow y, \bar{x} \& y\},
$$

реализуюшие линейные булевы функции $l_{n} u \bar{l}_{n}, n \geqslant 2$, состоят из стандартньх попарно непересекающихся блоков.

Стандартные блоки в [9] определялись как схемы, моделирующие одну из формул $(x \rightarrow y) \rightarrow(\bar{x} \& y)$ и $\overline{(\bar{x} \& y)} \&(x \rightarrow y)$, то есть представляли собой (в терминологии данной статьи) стандартные блоки для базиса $\{x \rightarrow y, \bar{x} \& y, \bar{x}\}$, не содержащие инверторов. Поскольку минимальная схема в базисе $\{x \rightarrow y, \bar{x} \& y\}$ является минимальной схемой в базисе $\{x \rightarrow y, \bar{x} \& y, \bar{x}\}$, теорема 3 сразу следует из теоремы 2 .

В заключение автор приносит глубокую благодарность своему научному руководителю, профессору Н. П. Редькину. 


\section{Список литературы}

1. Лупанов О. Б., Асимптотические оценки сложности управляющих систем. МГУ, Москва, 1984.

2. Редькин Н. П., Доказательство минимальности некоторых схем из функциональных элементов. Проблемы кибернетики (1970) 23, 83-101.

3. Редькин Н. П., О минимальной реализации линейной функции схемой из функциональных элементов. Кибернетика (1971), № 6 31-38.

4. Шкребела И. С., О сложности реализации линейных булевых функций схемами из функциональных элементов в базисе $\{x \rightarrow y, \bar{x}\}$. Дискретная математика (2003) 15, №4, 100-112.

5. Редькин Н. П., О минимальных и асимптотически минимальных схемах для некоторых индивидуальных булевых функций. В сб.: Материаль IX Международного семинара «Дискретная математика и ее приложения». Изд-во мех.-мат. ф-та МГУ им. М. В. Ломоносова, Москва, 2007, c. 11-19.

6. Ложкин С. А., О структуре минимальных схем в базисе $\{\&, \vee, \neg\}$, реализующих линейную функцию. В сб.: Труды V Международной конференции «Дискретные модели в теории управляюших систем». Изд. отдел ф-та ВМиК МГУ им. М. В. Ломоносова, Москва, 2003, с. 50-51.

7. Комбаров Ю. А., О минимальных реализациях линейных булевых функций. Дискретный анализ и исследование операџий (2012) 19, №3, 39-57.

8. Редькин Н. П., Дискретная математика. Физматлит, Москва, 2009.

9. Комбаров Ю. А., О минимальных реализациях линейных булевых функций схемами из функциональных элементов в базисе $\{x \rightarrow y, \bar{x} \& y\}$. В сб.: Tруды VIII Международной конференции «Дискретные модели в теории управляющих систем». МАКС Пресс, Москва, 2009, с. 145-149.

Статья поступила 19.11.2011. 